\title{
Fabrication of Microscale Tungsten Carbide Workpiece by New Centerless Grinding Method
}

\author{
Yufeng Fan
}

Additional information is available at the end of the chapter

http://dx.doi.org/10.5772/51405

\section{Introduction}

Recent years have seen the rapid increase in the demand for microscale components smaller than $100 \mu \mathrm{m}$ in diameter, such as micro machine parts, micromachining tools, micro pin gauges, medical catheters, and probes used in scanning tunneling microscope (STM) and semiconductor inspection. To meet this demand, many researchers have actively engaged in the development of new technology for fabricating such devices precisely and efficiently by non-traditional or mechanical machining methods.

Non-traditional machining has employed laser beam lithography and the focused ion beam method. Maruo and Ikuta [1], Yamaguchi et al. [2], and Nakai and Marutani [3] utilized laser beam lithography to fabricate 3D microscale photopolymer components including microscale cylindrical parts. Vasile et al.[4] developed a processing method for the sharpening of STM probes with a focused ion beam. Furthermore, electric discharge machining (EDM) technology is quite effective in micromachining, as seen, for example, in studies on wire EDM of minute electrodes by Heeren et al. [5] and Masuzawa et al. [6,7]. However, these non-traditional methods can only be applied to a limited set of materials, and problems involving machining efficiency and accuracy have not been resolved.

On the other hand, traditional mechanical machining methods, such as cutting and grinding, have also been employed in microscale fabrication. For example, Uehara et al. [8] studied electrolytic in-process dressing (ELID) cylindrical grinding of a micro-shaft, and Okano et al. [9] researched cylindrical grinding of a micro-cylinder. Yamagata and Higuchi [10] developed a four-axis controlled ultra-precision machine and conducted precision turning experiments on a stepped shaft. In these traditional mechanical methods, however, the workpiece is held at its end by a chuck or at both ends by two centers during machining operation. Consequently, it is difficult to perform high-efficiency, high-accuracy machining, 
especially on microscale cylindrical workpieces with a large aspect ratio because of the low stiffness of the workpiece support mechanism. Fortunately, these problems can be solved if a centerless grinding technique is employed since the workpiece can then be supported along its entire length on a regulating wheel and blade. However, in microscale machining by conventional centerless grinding, an extremely thin blade is required because the blade thickness must be smaller than the workpiece diameter so that the regulating wheel does not interfere with the blade. This necessitates the installation of a costly blade and significantly reduces the stiffness of the workpiece support mechanism. In addition, because of the extremely low weight, the microscale workpiece springs from the blade easily during grinding due to the surface tension of the grinding fluid adhering to the lifting regulating wheel circumference surface. This phenomenon is called "spinning" [11], and causes the grinding operation to fail. However, as will be explained below, these problems would be overcome by employing the ultrasonic-shoe centerless grinding technique developed by the present authors [12-15] in microscale fabrication.

\section{Ultrasonic vibration shoe centerless grinding method}

Fig. 1(a) illustrates the principle of ultrasonic-shoe centerless grinding where an ultrasonic shoe and a blade are used to support the workpiece and feed it toward the grinding wheel, instead of using a regulating wheel as in conventional centerless grinding (see Fig. 1(b)). In the former case (see Fig. 1(a)), an ultrasonic vibration shoe supports the workpiece and feed it towards the grinding wheel. The rotational speed of the workpiece is controlled by the elliptic motion of the shoe end face. Whereas in the latter case (see Fig. 1(b)), the conventional centerless grinding method includes three basic elements: grinding wheel, regulating wheel and blade. A regulating wheel supports the workpiece together with a blade and to feed the workpiece towards the grinding wheel. The rotational speed of the workpiece is controlled by the rotation of the regulating wheel.

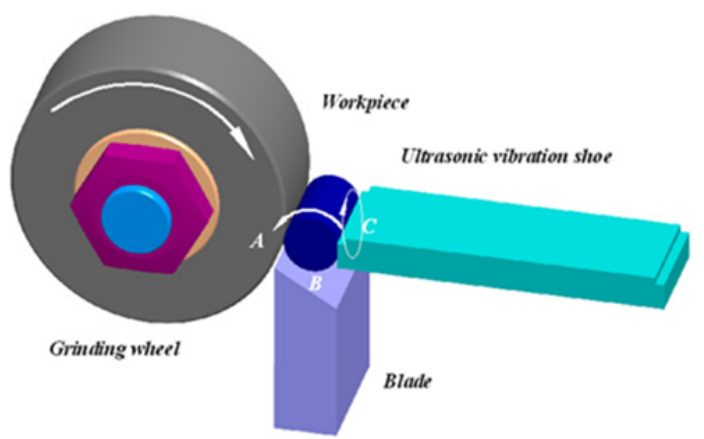

(a)

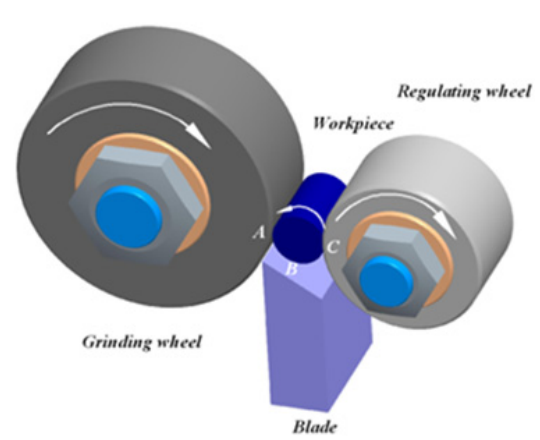

(b)

Figure 1. Illustrations of the new centerless grinding with ultrasonic shoe (a) and conventional centerless grinding with regulating wheel (b) 
The Fig.2 shows the detail principle of ultrasonic vibration shoe centerless grinding. The workpiece is supported by an ultrasonic elliptic-vibration shoe together with a blade, and it is fed towards the grinding wheel by the shoe. When two alternative current (AC) signals (over $20 \mathrm{kHz}$ ) with a phase difference of $\Psi$, generated by a wave function generator, are applied to the PZT after being amplified by means of power amplifiers, the bending and longitudinal ultrasonic vibrations are excited simultaneously. The synthesis of vibration displacements in the two directions creates an elliptic motion on the end face of the metal elastic plate.

Consequently, the rotation of workpiece is controlled by the friction force between the workpiece and the shoe so that the peripheral speed of the workpiece is the same as the bending vibration speed on the shoe end face. The speed varies with the variation of the voltage. In addition, the geometrical arrangements of workpiece such as the shoe tilt angle $\beta$, the workpiece center height angle $\alpha$ over the grinding wheel center, and the blade angle $\phi$ can be adjusted to get the optimum geometrical arrangement in order to achieve the least roundness error.

Based on the processing principle described above (see Fig.2), a grinding apparatus was built as illustrated in Fig.3. The cylindrical workpiece is constrained between the ultrasonic shoe, the blade, and the grinding wheel. The shoe and the blade are fixed on their holders by using bolts. A fine feed mechanism consisting of a linear motion way, a ball screw, and the shoe holder is driven by a stepping motor to give the shoe a fine

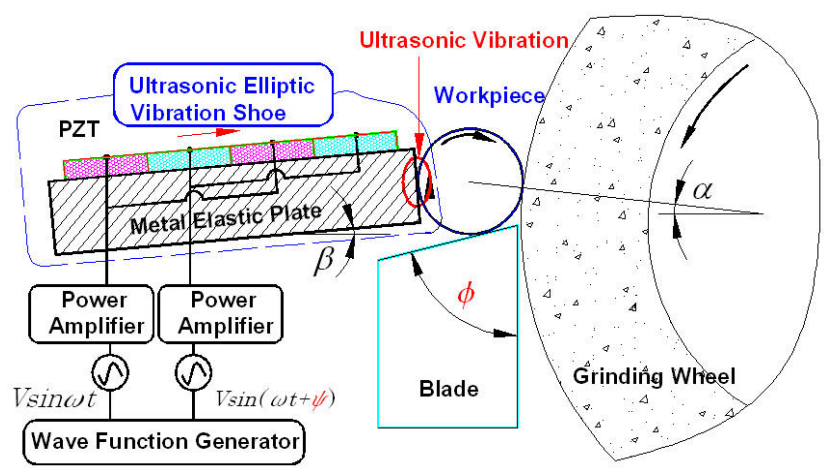

Figure 2. The detail principle of ultrasonic vibration shoe centerless grinding

Motion forward and backward on to the grinding wheel during grinding. The rotational speed of the workpiece is controlled by the elliptic motion of the shoe. Once the clockwise rotating workpiece interferes with the grinding wheel that is rotating counterclockwise at high speed, the workpiece is fed forward and grinding commences. As can be seen in Fig.1 (a), the gap between the lower right edge of the shoe and the top face of the blade should be smaller than the workpiece diameter; otherwise the workpiece would fall through the gap, causing the grinding operation to fail. Therefore, when grinding a microscale workpiece less than $100 \mu \mathrm{m}$ in diameter, the vertical position of the shoe must be adjusted carefully so that 
the gap is sufficiently small. To this end, a fine vertical position adjustment mechanism composed of a vertical motion guide, a ball screw, and a table, on which the fine feed mechanism is held, was constructed in order to adjust the gap by manipulating the ball screw. Moreover, a pre-load is applied to the shoe at its left end face along its longitudinal direction using a coil spring in order to prevent the shoe from breaking due to resonance.

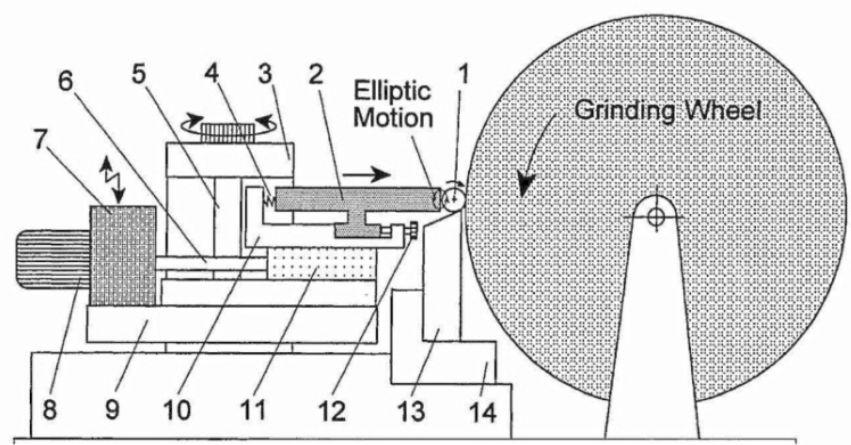

1.Workpiece 2.Ultrasonic shoe 3.Vertical motion guide 4.Pre-load spring 5.Ball screw for adjusting the vertical position of shoe 6.Ball screw for feeding the shoe 7.Gear head 8.Stepping motor 9.Table for holding fine feed mechanism 10.Shoe holder 11.Linear motion way 12.Bolt for fixing the shoe 13.Blade 14.Blade holder

Figure 3. Illustration of the new centerless grinding apparatus

\section{Design and construction of an ultrasonic shoe}

Fig.4 ( $\mathrm{a}$ and $\mathrm{b}$ ) shows the ultrasonic shoe structure and the principle of generation of ultrasonic elliptic motion on the shoe end face, respectively. As shown in Fig.4 (a), the shoe is constructed by bonding a piezoelectric ceramic device (PZT) having four separated electrodes on to a metal elastic body (stainless steel,SUS304). Applying two alternating current voltages (frequency $f$, amplitude $V_{p-p}$, and phase difference $\psi$ ) generated by amplifying two AC signals from a wave function generator with power amplifiers to the PZT induces simultaneous bending and longitudinal ultrasonic vibrations with amplitudes of several micrometers(see Fig.4(b)). The synthesis of vibration displacements, $U_{B}$ and $U_{L}$, in the two directions creates an elliptic motion on the end face of the metal elastic body. Consequently, the rotation of the workpiece is controlled by the friction force between the workpiece and the shoe (see Fig.1 (a)), and the peripheral speed, $V_{w}$, of the workpiece is thus the same as the bending vibration speed, $V_{S}\left(=f \times U_{B}\right)$, on the shoe end face.

Thus, it is essential that the two vibration modes, i.e., bending vibration (B-mode) and longitudinal vibration (L-mode), of the shoe must be induced simultaneously at the same frequency in order to generate an elliptic motion on the shoe end face. The present authors have pointed out in previous studies [12-15] that a combination of an even-ordered B-mode (i.e.,B2, B4,B6, B8, etc.) and an odd-ordered L-mode(i.e.,L1,L3,L5,L7,etc.), where the sole common node for the B-mode and L-mode is located at its central position, should be selected so that the ultrasonic vibrations of the shoe would not be restricted when held at 
the common node on the shoe holder. In addition, the simpler the vibration mode is, the easier the excitation of the shoe. From this viewpoint, a combination of L1 and B2 modes is desired. However, when the shoe is treated as a plate of length 1 with a uniform crosssection of width $b$ and thickness $t$ for simplicity, the precondition that the frequency of the rth L-mode must be the same as that of nth B-mode yields the following relationship between $l$ and $t[16]$ :

$$
l=\frac{(2 n+1)^{2} \pi t}{8 \sqrt{3} r}
$$

Eq. (1) gives the relationship $l=5.7 \mathrm{t}$ for the $\mathrm{L} 1 \mathrm{~B} 2(\mathrm{r}=1, \mathrm{n}=2)$ combination, but the relationship $l=18.4 \mathrm{t}$ for the L1B4 $(\mathrm{r}=1, \mathrm{n}=4)$ combination. This suggests that a thin type shoe, the vibration excitation of which can be more easily compared with others, can be constructed based on the L1B4 combination. Thus, the L1B4 combination was selected as the ultrasonic shoe.

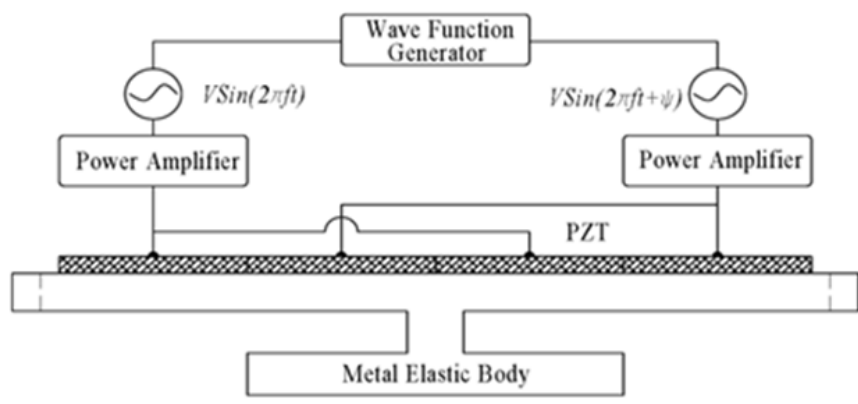

(a) Shoe structure and power application method

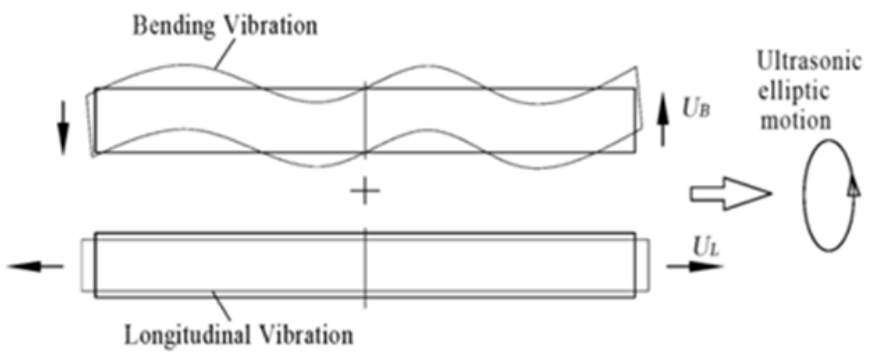

(b)Generation principle of elliptic motion

Figure 4. Structure and operating principle of the ultrasonic elliptic vibration shoe

Based on the discussion above, the structure proposed is shown in detail in Fig.5. A Tshaped extrusion is located at the center of the shoe via which the shoe can be fixed on its holder by bolts. Four separate electrodes are distributed on the PZT based on the B4 mode. The dimensions of the shoe are then determined by FEM analysis followed by impedance measurement to be described later. 


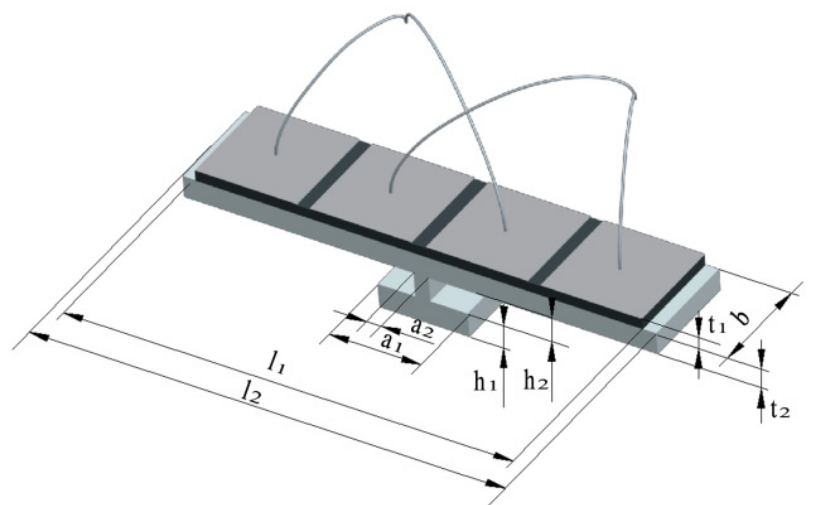

Figure 5. Fig.5 Detailed structure of the ultrasonic shoe

\begin{tabular}{l|l|l|l|l|l|l|l|l}
\hline \hline$b$ & $t_{1}$ & $t_{2}$ & $l_{1}$ & $l_{2}$ & $h_{1}$ & $h_{2}$ & $a_{1}$ & $a_{2}$ \\
\hline 20 & 2 & 4 & 88.6 & & 3 & 20 & 20 & 5 \\
\hline \hline
\end{tabular}

Table 1. The dimensions of shoe designed ( $\mathrm{mm})$
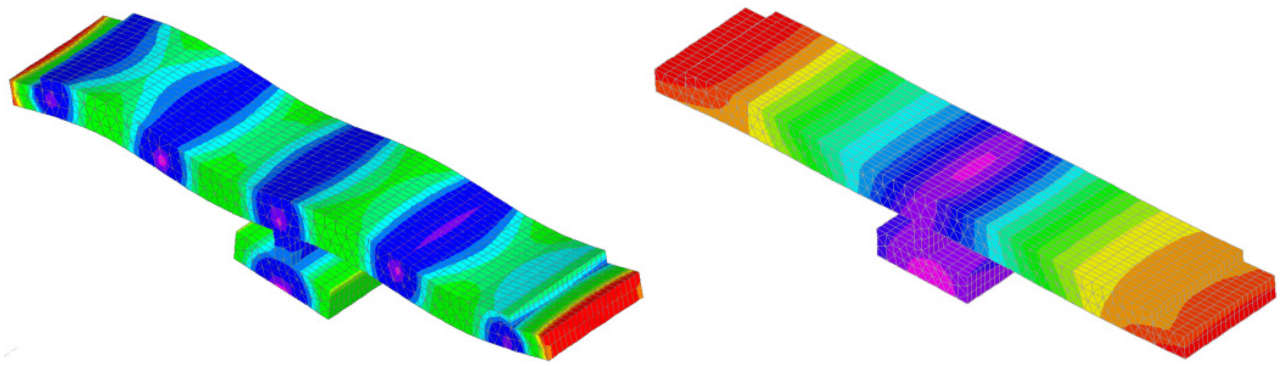

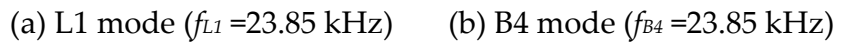

Figure 6. L1 and B4 modes obtained by FEM analysis

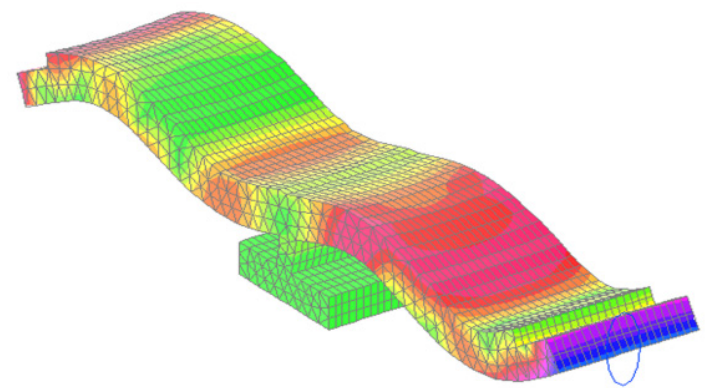

Figure 7. Elliptic motion predicted by FRA (frequency response analysis) 
With the exception of the length of the metal elastic body, $l_{2}$, all dimensions were determined (see Table1) by taking into consideration the space available for installation of the proposed shoe on the existing centerless grinder. Dimension $l_{2}$ was first predicted by finite element method (FEM) analysis under the condition $f_{L 1}$ (frequency of L1 mode) $=f_{B A}$ (frequency of B4 mode). Fig.6 ( $\mathrm{a}$ and b) shows, respectively, the L1 and B4 modes of a shoe $\left(l_{2}=96.95 \mathrm{~mm}\right)$ obtained by FEM analysis for $f_{L 1}=f_{B 4}=23.85 \mathrm{kHz}$. In order to confirm the generation of elliptic motion on the shoe end face having the FEM predicted dimension $l_{2}$ $=96.95 \mathrm{~mm}$, a frequency response analysis (FRA) was carried out using piezoelectric device analysis software. Fig.7 shows the FRA results obtained for $V_{\mathrm{p}-\mathrm{p}}=50 \mathrm{~V}, f=23.90 \mathrm{kHz}$, and $\psi$ $=90^{\circ}$. Clearly, an elliptic motion occurs on the end face of the shoe.

As predicted by FEM and FRA above, $l_{2}$ must be $96.95 \mathrm{~mm}$ in order for $f_{L 1}$ to equal $f_{B 4}$ and for an elliptic motion to be generated on the shoe end face. However, it is foreseen that the actual values of $f_{L 1}$ and $f_{B 4}$ would not agree with the predicted values due to dimensional errors associated with the metal elastic body and the PZT used. Thus, three shoes with different values of $l_{2}$, namely $96.45,96.95$, and $97.45 \mathrm{~mm}$, were constructed based on the FEM and FRA results. One of these values was selected after the shoes' actual frequencies $f_{L 1}$ and $f_{B 4}$ were obtained by measuring their impedance characteristics. Fig. 8 shows a photograph of a designed and constructed ultrasonic shoe. The shoe surface was coated with a waterproofing layer in order to protect against the grinding fluid during grinding. Further, the friction coefficient between the shoe and the workpiece should be large enough to prevent the workpiece from slipping on the shoe end face. Thus, a thin rubber $(0.5 \mathrm{~mm}$ in thickness) sheet made of the same materials that used in conventional regulating wheels was prepared and attached to the end face of the shoe.

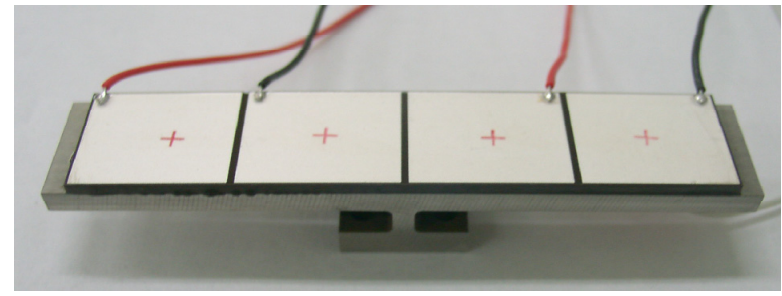

Figure 8. Photograph of a fabricated shoe

An impedance analyzer was used for investigating the impedance characteristics of the shoes. The results obtained for the shoe having an $l 2$ of $96.95 \mathrm{~mm}$ are shown in Fig.9 (a and b) for the L1 and B4 modes, respectively. Clearly, the impedances for the B4 and L1 modes reach their minima at the frequencies of 24.13 and $24.01 \mathrm{kHz}$, respectively, indicating that the respective resonant frequencies for the $\mathrm{L} 1$ and $\mathrm{B} 4$ modes are $f_{B 4}=24.13 \mathrm{kHz}$ and $f_{L 1}$ $=24.01 \mathrm{kHz}$. The impedances for the two modes reach their maxima at 24.20 and $24.22 \mathrm{kHz}$, respectively, meaning the power consumption would be least when the AC voltages applied at these frequencies. This is referred to as the anti-resonance effect $[17,18]$. The measured $f_{L 1}$ and $f_{B 4}$ are plotted against $l_{2}$ (Fig.10). It can be seen that $f_{L 1}$ comes closest to $f_{B 4}$ at $l_{2}=96.45 \mathrm{~mm}$. Thus, $l_{2}$ was determined to be $96.45 \mathrm{~mm}$. 


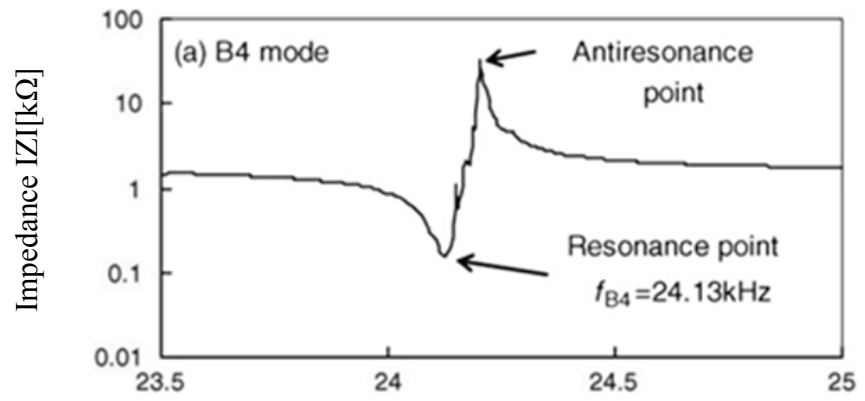

Frequency $f[\mathrm{kHz}]$

(a)Impedance characteristics of B4 mode

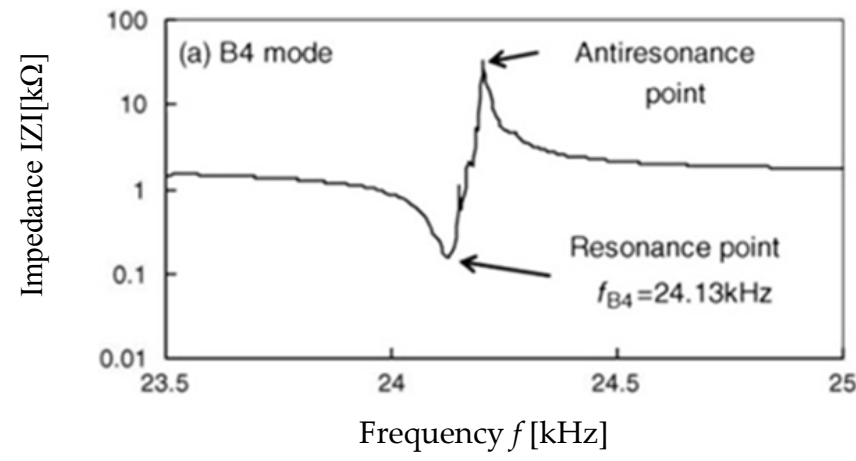

(b)Impedance characteristics of L1 mode

Figure 9. Impedance characteristics of the shoe

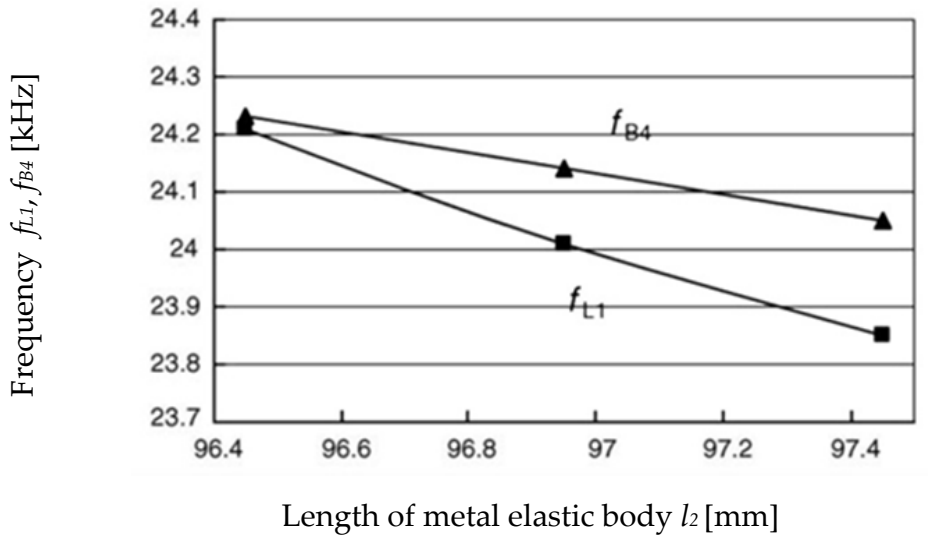

Figure 10. Measured frequencies of two modes 


\section{Performance of the apparatus constructed}

\subsection{Method of measuring the ultrasonic elliptic vibration}

The elliptic motion of the shoe end face under various applied voltages (amplitudes, frequencies and phase differences) is investigated using a measuring system composed of two laser Doppler vibrometers (Ono Sokki Co., Ltd., LV-1610) equipped with the respective sensor heads, a vector conversion unit (Ono Sokki Co., Ltd.,), and a multi-purpose FFT(Fast Fourier Transform) analyzer (Ono Sokki Co., Ltd., CF-5220), as shown in Fig.11.

The shoe is bolted at its center (the common node for L1 and B4 mode) on the holder in order not to restrict the ultrasonic vibration. A preload is then applied to the shoe using a coil spring in order to prevent the PZT from breaking due to resonance. Two AC signals generated by a wave function generator (NF Corporation, WF1994) are applied to the PZT after being amplified by two power amplifiers (NF Corporation, 4010). During measurement, the two laser beams from the respective heads are focused at the same point near the shoe end face. The signals from the laser Doppler vibrometers are then input to the vector conversion unit for synthesis and are recorder with a digital oscilloscope (Iwatsu Co., Ltd., LT364L). The AC signal is changed by various voltages, phase differences and frequencies. From the digital oscilloscope, the trace of ultrasonic vibration will be obtained based on the different input parameters, and the relationship between the input parameters and the vibration will be clarified.

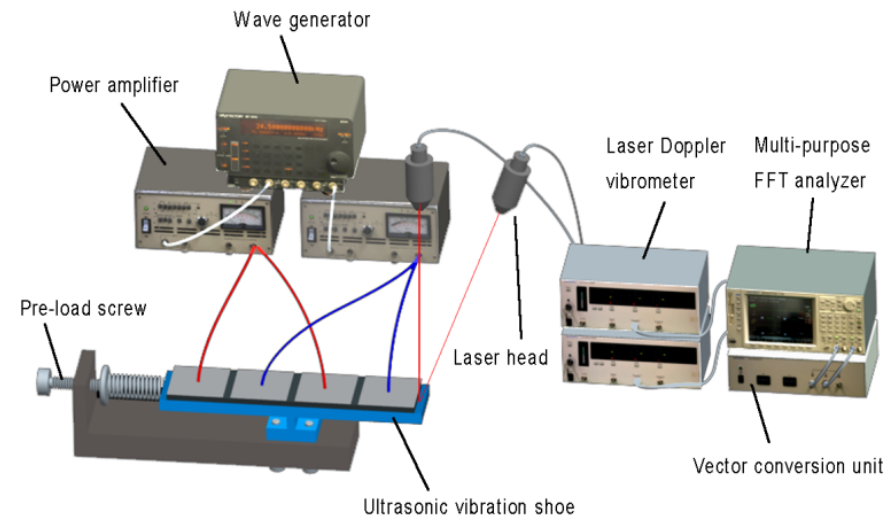

Figure 11. Method of measuring the ultrasonic elliptic vibration

The shoe is bolted at its center (the common node for L1 and B4 mode) on the holder in order not to restrict the ultrasonic vibration. A preload is then applied to the shoe using a coil spring in order to prevent the PZT from breaking due to resonance.

Two AC signals generated by a wave function generator (NF Corporation, WF1994) are applied to the PZT after being amplified by two power amplifiers (NF Corporation, 4010). During measurement, the two laser beams from the respective heads are focused at the same 
point near the shoe end face. The signals from the laser Doppler vibrometers are then input to the vector conversion unit for synthesis and are recorder with a digital oscilloscope (Iwatsu Co., Ltd., LT364L). The AC signal is changed by various voltages, phase differences and frequencies. From the digital oscilloscope, the trace of ultrasonic vibration will be obtained based on the different input parameters, and the relationship between the input parameters and the vibration will be clarified.

Fig.12 shows the measured results of the point on the end face with various parameters.

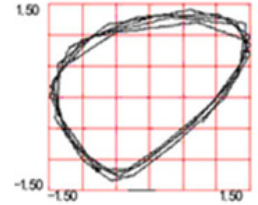

$f=24.2 \mathrm{kHz}$

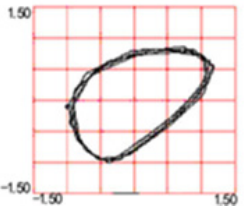

$f=24.3 \mathrm{kHz}$

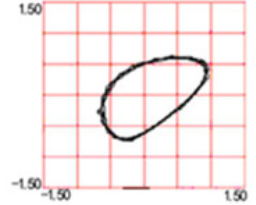

$f=24.4 \mathrm{kHz}$

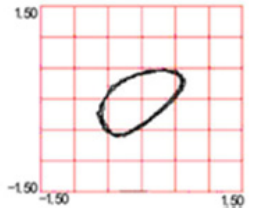

$f=24.5 \mathrm{kHz}$

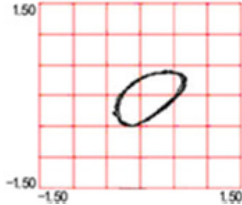

$f=24.6 \mathrm{kHz}$

(a) Elliptic vibration for various frequencies $\left(V_{p-p}=100 \mathrm{~V}, \psi=90^{\circ}\right)$
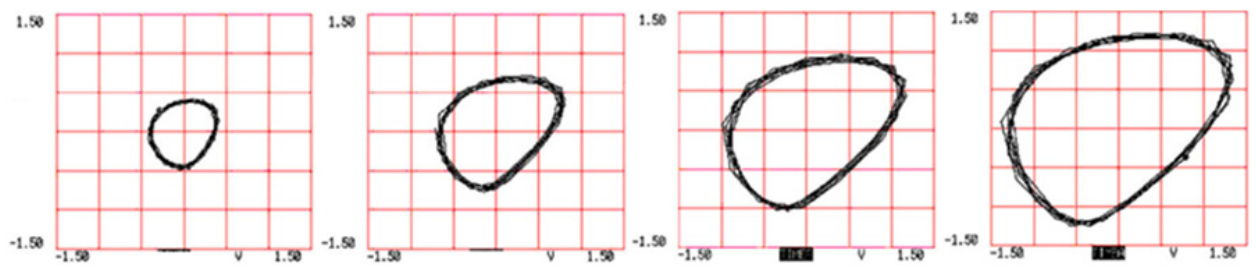

$\mathrm{V}_{\mathrm{p}-\mathrm{p}}=50 \mathrm{~V}$

$\mathrm{V}_{\mathrm{p}-\mathrm{p}}=100 \mathrm{~V}$

$\mathrm{V}_{\mathrm{p}-\mathrm{p}}=150 \mathrm{~V}$

$\mathrm{V}_{\mathrm{p}-\mathrm{p}}=200 \mathrm{~V}$

(b) Elliptic vibration for various applied voltages $\left(f=24.3 \mathrm{kHz}, \psi=90^{\circ}\right)$

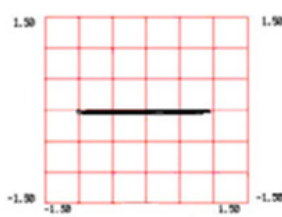

$\psi=0^{\circ}$

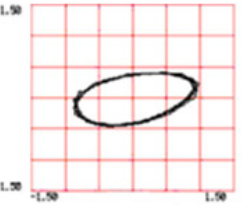

$\psi=45^{\circ}$

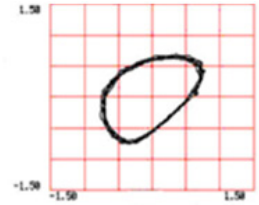

$\psi=90^{\circ}$
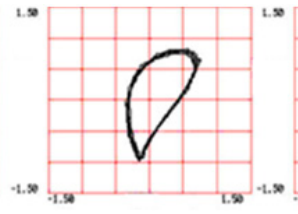

$\psi=135^{\circ}$

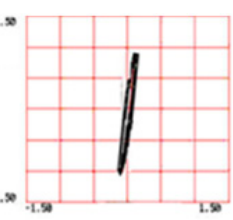

$\psi=180^{\circ}$

(c) Elliptic vibration for various phase differences $\left(V_{p-p}=100 \mathrm{~V}, f=24.3 \mathrm{kHz}\right)$

Figure 12. Measured results of the point on the end face with various parameters

\subsection{Rotational motion control tests of the workpiece}

In the ultrasonic vibration shoe centerless grinding method, it is crucial to precisely control the workpiece rotational speed by the elliptic motion of the end face of shoe in order to achieve high-precision grinding. Therefore, a evaluating involving the rotational control of a cylindrical workpiece using the produced ultrasonic vibration shoe was conducted on an apparatus specially built in house, as shown in Fig.13. 
In the apparatus, a wheel mounted on a spindle is driven rotationally by a motor and plays the role of the grinding wheel. The ultrasonic vibration shoe is bolted on its holder and then held on a small 2-axis dynamometer (Kistler Co., Ltd., 9876) installed on a linear motion guide. A thin rubber sheet $(0.5 \mathrm{~mm}$ in thickness) of the same material as that of a conventional regulating wheel (A120R) was made and attached to the shoe end face so that the friction coefficient between the shoe and the workpiece is large enough to prevent the workpiece from slipping on the shoe end face. The workpiece is fed toward the wheel by the shoe, which is carried forward by manipulating the shoe feed bolt. The normal contact force and the friction force between the rotating workpiece and wheel correspond to the normal and tangential grinding forces, respectively. In the test, the dynamometer was used to set up the force, and the same wave function generator and power amplifiers as used in the elliptic motion measurement were employed to apply the AC voltage to the PZT. The workpiece rotational speed is obtained by recording the motion of the rotating workpiece end face, on which a circular mark was created, suing a digital video camera. The video images are then stored in a computer for analysis using animated image processing software (Deigimo Co., Ltd., Swallow2001 DV). Pin-shaped rods (SK4) of 5mm in diameter and $15 \mathrm{~mm}$ in length were used as the workpieces. In addition, $V_{p-p}$ was set in the range of $20-200 \mathrm{~V}$ while the voltage frequency and the phase difference were fixed at $f=24.3 \mathrm{kHz}$ and $\psi=90^{\circ}$, respectively.

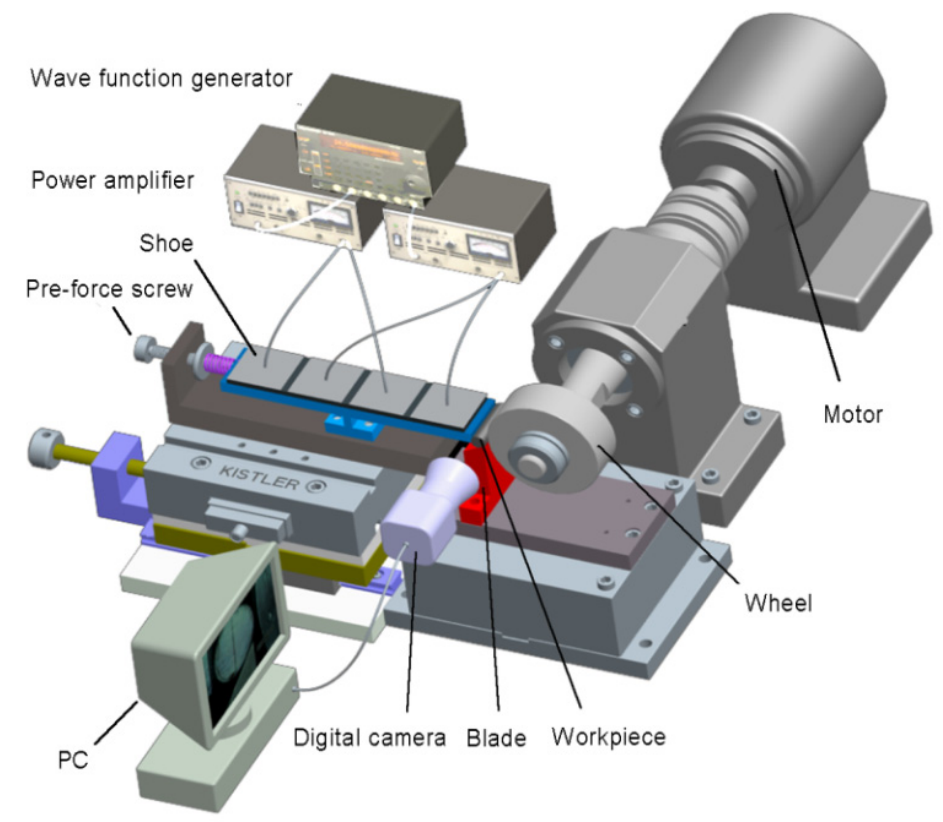

Figure 13. Evaluation apparatus for the shoe

Fig.14 shows a series of video images of the workpiece end face taken every 0.033 s with a camera capable of taking 30 pictures per second. The workpiece rotational speed $n_{w}$ can thus be calculated as follows: 


$$
n_{w}=\frac{\sum_{i=1}^{N} n_{w i}}{N}
$$

where $\mathrm{n}_{\mathrm{wi}}=\left(\beta_{i+1}-\beta_{i}\right) /\left(t_{i+1}-t_{i}\right), \mathrm{i}=1,2, \ldots, \mathrm{N}$.

Fig.15 shows the relationship obtained between $n_{w}$ and $V p-p$. Clearly, $n_{w}$ increases linearly with $V_{p-p}$. This is in close agreement with the prediction described above, and indicates that the workpiece rotation speed can be precisely controlled by the elliptic motion of the shoe.
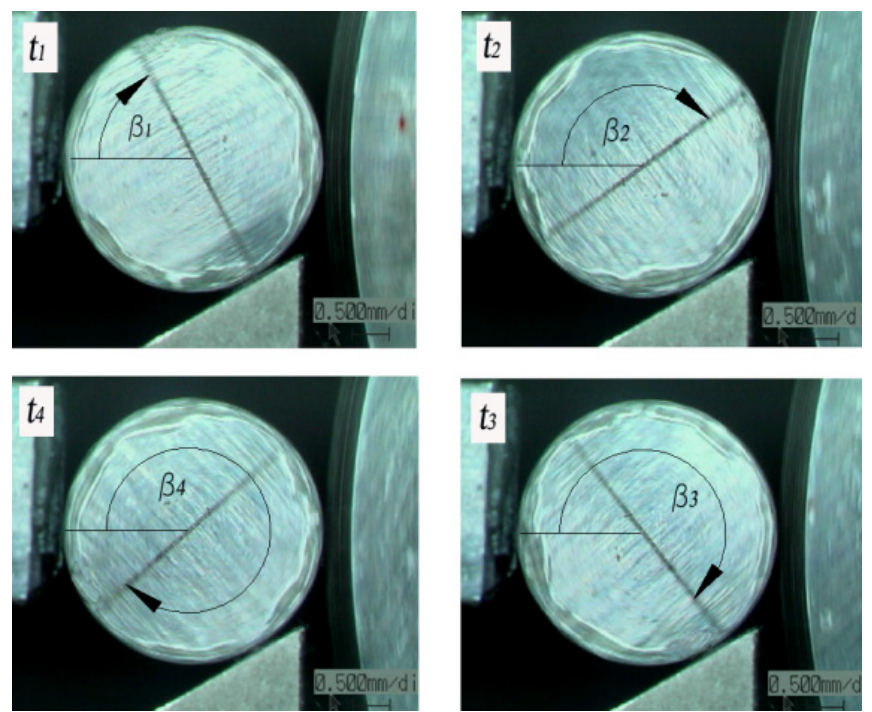

Figure 14. Video images of the rotating workpiece
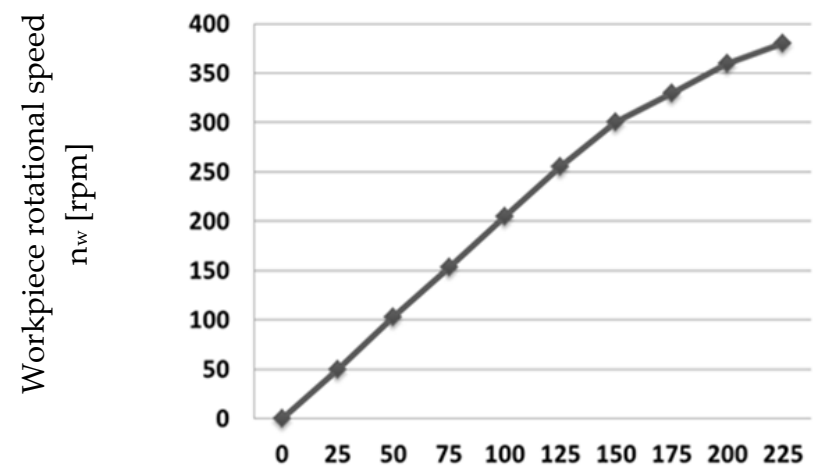

Voltage $\mathrm{V}_{\mathrm{p}-\mathrm{p}}[\mathrm{V}]$

Figure 15. Relationship between the workpiece rotational speed and the applied voltage 


\section{Fabrication of micro-part of tungsten carbide}

\subsection{The modification of experimental grinder}

In order to confirm the validity of the proposed new method, fabrication of micro-part of tungsten carbide will be carried out. The grinder is modified by the conventional grinder of $\mu$ micron grinder MIC-150, the product of $\mu$ micron Corp. The regulating wheel unit will be uninstalled and a fine feed unit, which is composed of a fine feed table and stepping motor, will be installed. In the experimental grinder, for the finish grinding of micro parts with sizes of less than $1 \mathrm{~mm}$ in diameter, the depth of cut must be less than $1 \mu \mathrm{m}$ in order to make the grinding force small. The fine feed and fine adjustment unit is shown in Fig.16 ( $a$ and $b$ ). The shoe can be fine adjusted in $\mathrm{Z}$ direction by handing the fine adjustment screw. The adjustment component can be locked when the height of shoe is adjusted to an appreciable position by operating the lock handle on the back of the unit, as shown in Fig.16 (b). The fine feed and fine adjustment unit can be rotated in $X Y$ by surrounding the rotating pin, and then fixed the unit by locking other three fixed screw bolts. A fine feed unit composed of a shoe holder, a linear guide, a ball screw and a stepping motor has been designed and produced that carries the shoe toward the grinding wheel at a feed rate of less than $1 \mu \mathrm{m}$. A pre-load is then applied to the shoe at its left end face in its longitudinal direction using a coil spring in order to prevent the shoe from breaking due to resonance and is fixed by the screw at its right face of shoe foot.

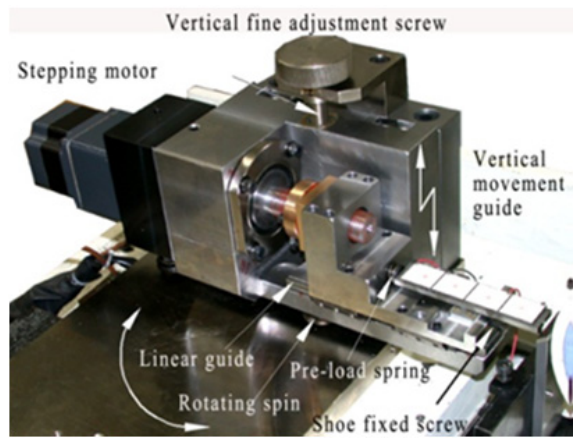

(a)

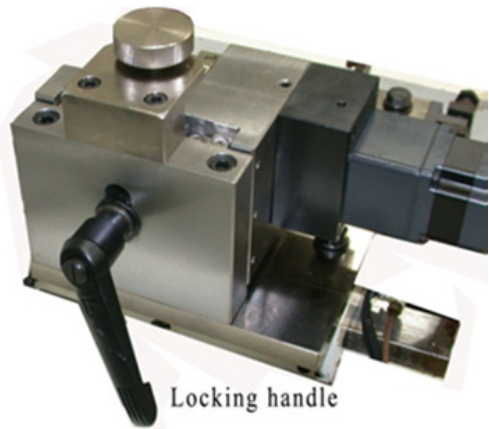

(b)

Figure 16. Fine feed and fine adjustment unit

\subsection{Grinding experiments}

The grinder installed fine feed and adjustment mechanisms was used to grinding microscale cylindrical workpiece, its aim is to verify the feasibility of micro-scale fabrication by ultrasonic-shoe centerless grinding technique, and to confirm the performance of the constructed experimental apparatus in actual grinding operations. The tungsten carbide steel cylindrical workpiece used in grinding is shown in Fig.17, 0.6mm in diameter and $15 \mathrm{~mm}$ in length. The photo of grinder is shown in Fig.18 and Fig.19 shows a main portion of the experimental setup. The experimental conditions are listed as in Table 2. 


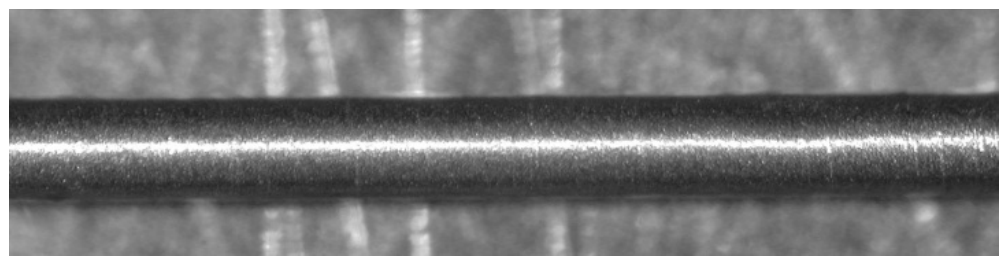

Figure 17. Original tungsten carbide steel cylindrical workpiece (D0.6mm $\times$ L15mm)

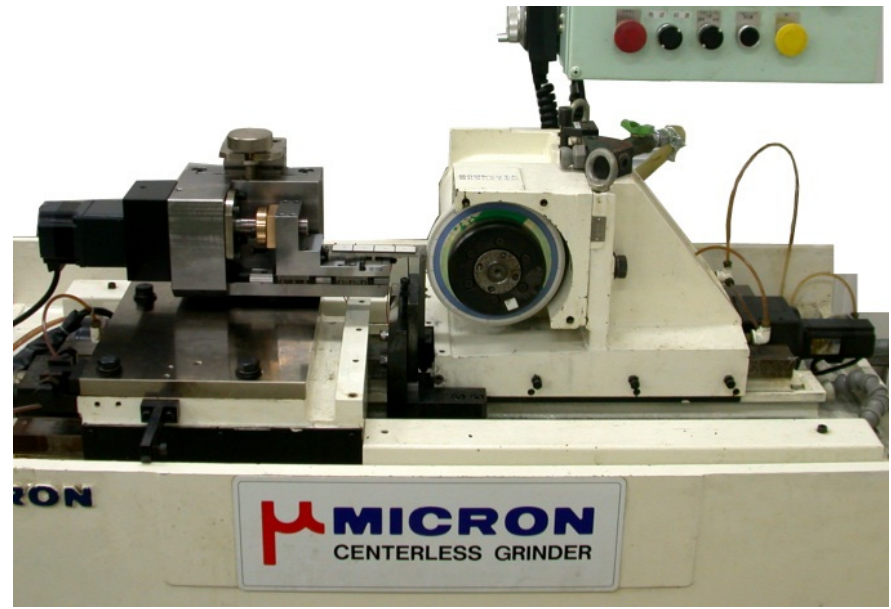

Figure 18. Photo of grinder installed a fine feed and adjustment mechanisms

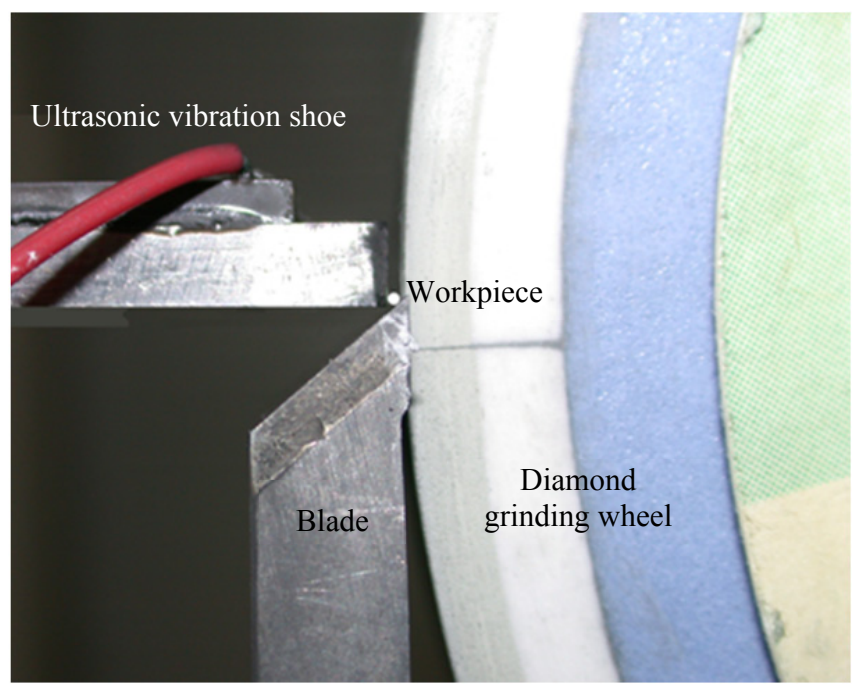

Figure 19. Experimental setup for the grinding test 
The grinding test was performed as follows: first, the gap between the right-down edge of the shoe and the top face of the blade was set up carefully using the fine vertical position adjustment mechanism so that the gap is smaller than the final diameter of the workpiece after grinding. Next, the grinding wheel was moved toward the blade and stopped soon after they have interfered with each other. Subsequently, the shoe was carried forward to feed the workpiece toward the grinding wheel at a feed rate of $V_{f}=0.1 \mathrm{~mm} / \mathrm{min}$ and ground under the grinding conditions listed in Table 2. The grinding operation was finished once the given stroke removal and spark out had been completed and the shoe had been then retracted from the grinding wheel.

\begin{tabular}{|c|c|c|c|c|}
\hline Grinding wheel & \multicolumn{4}{|c|}{ SD2000150×20×76.2,1A1 } \\
\hline Workpiece & \multicolumn{4}{|c|}{ Tungsten carbide steel $\phi 0.6 \times$ L15 } \\
\hline Coolant & \multicolumn{4}{|c|}{ Solution type } \\
\hline \multirow{7}{*}{$\begin{array}{l}\text { Grinding } \\
\text { parameters }\end{array}$} & \multirow{3}{*}{$\begin{array}{l}\text { Input } \\
\text { Voltage }\end{array}$} & \multicolumn{2}{|c|}{ Amplitude } & $V_{\mathrm{p}-\mathrm{p}}=100 \mathrm{~V}$ \\
\hline & & \multicolumn{2}{|c|}{ Frequency } & $f=24.3 \mathrm{kHz}$ \\
\hline & & \multicolumn{2}{|c|}{ Phase difference } & $90^{\circ}$ \\
\hline & \multicolumn{2}{|c|}{ Grinding wheel speed } & \multicolumn{2}{|c|}{$V_{\mathrm{g}}=30 \mathrm{~m} / \mathrm{s}$} \\
\hline & \multicolumn{2}{|c|}{ Shoe feed rate } & \multicolumn{2}{|c|}{$V_{f}=0.1 \mathrm{~mm} / \mathrm{min}$} \\
\hline & \multicolumn{2}{|c|}{ Stock removal } & \multicolumn{2}{|c|}{$0.4-0.55 \mathrm{~mm}$} \\
\hline & \multicolumn{2}{|c|}{ Spark-out time } & \multicolumn{2}{|c|}{$3 \mathrm{sec}}$. \\
\hline \multirow{2}{*}{$\begin{array}{l}\text { Geometrical } \\
\text { conditions }\end{array}$} & \multicolumn{2}{|c|}{ Center height angle } & \multicolumn{2}{|c|}{$\gamma=7^{\circ}$} \\
\hline & \multicolumn{2}{|c|}{ Blade angle } & \multicolumn{2}{|c|}{$\phi=60^{\circ}$} \\
\hline
\end{tabular}

Table 2. Conditions of grinding test

Fig.20 shows the SEM picture of the ground workpieces. Obviously, the cylindrical workpiece having an original diameter of $0.6 \mathrm{~mm}$ slimed down to one having dimension of $60 \mu \mathrm{m}$ in diameter, the aspect ratio of which is over 250. This result demonstrated that the constructed apparatus performed well even in actual micro-scale machining, and that microscale fabrication by ultrasonic-shoe centerless grinding technique is feasible. 


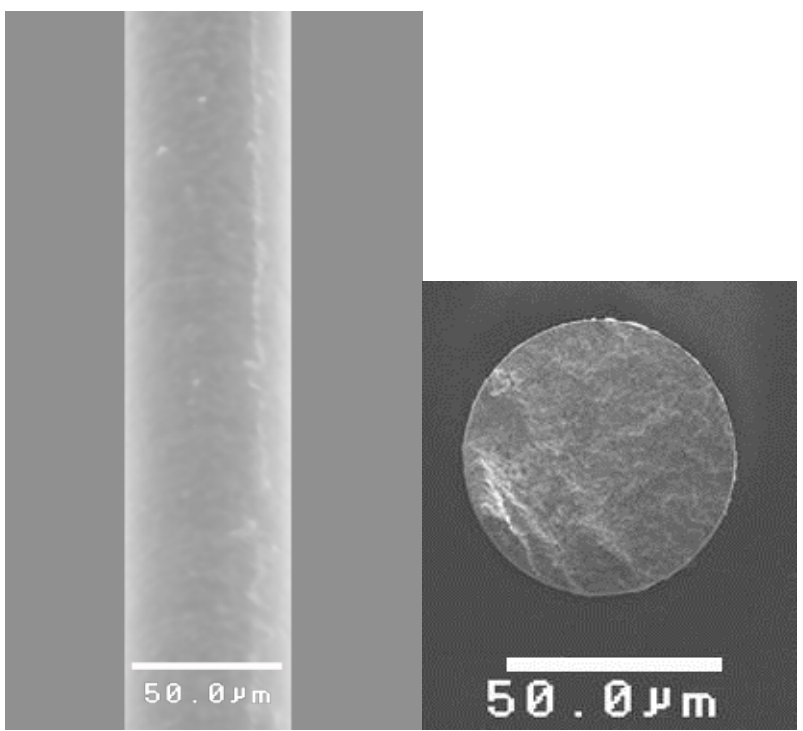

Figure 20. SEM image of the ground tungsten carbide steel workpiece

\section{Conclusions}

Using ultrasonic vibration shoe centerless grinding technique to fabricate microscale tungsten carbide steel cylindrical workpiece was investigated. An experimental apparatus was modified by installing fine feed and adjustment mechanisms, and the ultrasonic vibration shoe used in experimental grinding was designed and made especially. The tungsten carbide steel cylindrical workpiece with $0.6 \mathrm{~mm}$ in the original diameter and $15 \mathrm{~mm}$ in the length was ground with a diamond grinding wheel. As a result, a microscale tungsten carbide steel cylindrical workpiece of around $60 \mu \mathrm{m}$ in diameter and $15 \mathrm{~mm}$ in length, the aspect ratio of which was over 250 . The validity of the new microscale tungsten carbide steel cylindrical workpiece using ultrasonic vibration shoe centerless grinding technique is confirmed.

For a new microscale tungsten carbide steel cylindrical workpiece technology, it is further required to investigate influencing factors, such as the workpiece geometrical arrangement, and ultrasonic vibration amplitude how to affect the machining accuracy, i.e., workpiece roundness. The more fine adjustment mechanism and the measurement rig suitable for micro-scale workpiece less than $100 \mathrm{~m}$ also be designed and made.

Future work will focus on further developing this new technique in terms of the influence of grinding conditions, such as the workpiece geometrical configuration $(\phi, \gamma)$, on machining accuracy,i.e., workpiece roundness. To this end, it is essential to first develop a roundness measurement method for microscale components less than $100 \mu \mathrm{m}$ in diameter since there is, at present, no commercially available measurement rig suitable for such microscale components. This work will be detailed in a future report. 


\section{Author details}

Yufeng Fan

School of Mechanical \& Automotive Engineering Zhejiang, University of Science and Technology, Hangzhou, China

\section{References}

[1] Maruo S, Ikuta K. Two-photon micro stereolithography with submicron resolutionfabrication of a freely movable mechanism. In: Eighth international conference on rapid prototyping. 2000. p. 201.

[2] Yamaguchi K, Nakamoto T, Abraha P, Karyawan, Ito A. Manufacturing of microstructure using ultraviolet ray photoactive resin (3rdReport, Beam shape and hardening characteristics in focused beam drawing method). Trans Jpn Soc Mech Eng Ser C 1995;61(581):304.

[3] Nakai T, Marutani Y. Fabrication of resin model using ultraviolet laser. J Jpn Soc Technol Plast 1988; 29(335):1249.

[4] Vasile MJ, Grigg DA, Griffith JE, Fizgerald EA, Russell PE. Scanning probe tips formed by focused ion beams. Rev Sci Instrum 1991; 62(9):2167.

[5] Heeren P-H, Reynaerts D, Van Brussel H, Beuret C, Larsson O, Bertholds A. Microstructuring of silicon by electro-discharge machining (EDM)-part II. Appl Sens Actuators 1997;A61:379.

[6] Masuzawa T, Fujino M, Kobayashi K. Wire electro-discharge grinding for micro machining. Ann CIRP 1985;34(1):431.

[7] Masuzawa T, Fujino M. A process for manufacturing very fine pin tools. SME Technical Paper, MS90;1990. p.307/1-11.

[8] Uehara Y, etal. Development of small tool by micro fabrication system applying ELID grinding technique. In: Initiatives of precision engineering at the beginning of millennium, JSPE2001:10.

[9] Okano K, WaidaT, Suto T, Mizuno J, Kobayashi T. Micro-grinding of micromachine parts. In: Proceedings of international conference on abrasive technology. 1993. p.100.

[10] Yamagata $Y$, Higuchi T. Three-dimensional micro fabrication by precision cutting technique. J JSPE 1995; 61(10):1361.

[11] Hashimoto F. Effects of friction and wear characteristics of regulating wheel on centerless grinding. SME Technical Papers, MR99-226; 1999. p.1-10.

[12] Wu Y, Fan Y, Kato M, Wang J, Syoji K, Kuriyagawa T. A new centerless grinding technique without employing a regulating wheel. Key Eng Mater 2003; 238-239: 355360.

[13] Fan Y, Wu Y, Kato M, Tachibana T, Syoji K, Kuriyagawa T. Design of an ultrasonic elliptic-vibration shoe and its performance in ultrasonic elliptic-vibration-shoe centerless grinding. JSME Int J Ser C 2004;47(1):43-51. 
[14] Wu Y, Fan Y, Kato M, Kuriyagawa T, Syoji K, Tachibana T. Determination of an optimum geometrical arrangement of workpiece in the ultrasonic elliptic-vibration shoe centerless grinding. Key Eng Mater 2004; 257-258:495-500.

[15] Wu Y, Fan Y, Kato M, Kuriyagawa T, Syoji K, Tachibana T. Development of an ultrasonic elliptic vibration shoe centerless grinding technique. J Mater Process Technol 2004;155-156:1780-7.

[16] Ueha S, Tomikawa Y. New ultrasonic motor. Tokyo: Sougoudenshi Publications; 1991 [in Japanese].

[17] Kenjo N, Yubita S. Primer of ultrasonic motor. Tokyo: Sougoudenshi Publications; 1991[in Japanese].

[18] Piezoelectric ceramics technical handbook. Tokyo: Fuji Ceramics;1998[in Japanese]. 